\section{A Generalized Omega-Technique for Molecular Orbital Calculations}

Francisco M. Fernández and Eduardo A. Castro

Instituto de Investigaciones Fisicoquimicas Teóricas y Aplicadas, Sección Qu ìmica Teórica, La Plata 1900, Argentina

Z. Naturforsch. 34a, 1365-1368 (1979);

received April 30, 1979

A derivation of the omega-like methods to calculate electronic molecular structures is given. The derivation is based on the Hartree-Fock formalism and shows in a natural way how the omega-like methods follows through the retention of certain two-electron repulsion integrals and the neglection of other ones. A generalized omega-technique is proposed where both diagonal and non-diagonal Hamiltonian matrix elements depend on atomic charges and bond orders. Some numerical examples are presented for the calculation of bond lengths, ionization potentials and $\mathrm{Vl} \leftarrow \mathrm{N}$ electronic transitions.

\section{Introduction}

Several semi-empirical pi-electron calculations which employ different variants of the PariserParr-Pople (PPP) method $[1,2]$ have been carried out for conjugated hydrocarbon systems. The omega-technique [3] and various closely related methods $[4-10]$ constitute a subset of those semiempirical methods. The omega-technique is one of the simplest methods of taking into account the charge distribution in LCAO-MO calculations. It consists of modifying the Coulomb integral $\alpha_{\mu}$ at each centre by the pi-electronic density $q_{\mu}$ on that centre according to the relation

$$
\alpha_{\mu}=\alpha+\left(1-q_{\mu}\right) \omega \beta,
$$

where $\omega$ is an empirically evaluated dimensionless parameter with a generally accepted value of 1.4 [11] and $\beta$ is the resonance integral. A crude justification for the omega-technique was given by Flurry [12] by comparing (1) with the equations obtained in the PPP approximation. Mathur et al. [5] proposed a two-parameter omega-technique which amounts to an inclusion of the effect of nearest-neighbour electronic repulsion integrals into the conventional omega-technique. The pro-

Reprint requests to E. A. Castro, INIFTA, Sección Química Teórica, Sucursal 4, Casilla de Correo No. 16, La Plata 1900, Argentina. cedure outlined by Mathur et al. to obtain the equations for the Hamiltonian matrix elements is rather arbitrary. The authors start the derivation writting an equation analogous to (1) for $\beta_{\mu \nu}$ without any sound justification (see the discussion which follows Eq. (5) in Ref. [5]). An attempt has been made to follow a more rigorous derivation [13]. The IOC-omega-technique makes an approximate correction for the two centre electron repulsion integrals $[6,14]$ but the diagonal matrix elements do not involve an explicit dependence on atomic charges. The methods of Dorko et al. [7] and Huy and Forst [8] consider a dependence of the off-diagonal matrix elements $\beta_{\mu \nu}$ on the bond orders $p_{\mu \nu}$ but they ignore the dependence of the $\alpha_{\mu}$ terms on electron densities at the centres other than $\mu$. Despite of the semi-empirical nature of the omegalike methods, and although there exist differences among the various formulations, a common feature of them is that they work. The excellent correlations found between different theoretical and experimental quantities, show the usefullness of this kind of calculations (see Tables 1-3 of Ref. [4], and Tables 1-2 of Ref. [6]). The need for a theoretical basis of semi-empirical theories has been recently pointed out [15]. The immense utility of the semiempirical methods and their roots in tried and trusted chemical principles suggest that these approaches are somehow valid descriptions of chemical phenomena. The purpose of the present communication is to give a more rigorous derivation of the so-called omega-like methods, and to show how a generalized omega-technique may be formulated. Numerical examples are presented for the molecules chosen by Mathur et al. [5].

\section{Derivation of the Formulas for the Hamiltonian Matrix Elements}

The Hartree-Fock formulation is the most rigorous one to solve the problem of atomic and molecular atomic structures. Then we start the derivation from the expression for the Fock matrix elements

$$
F_{i j}=h_{i j}+J_{i j}-\frac{1}{2} K_{i j},
$$

where $R_{k l}$ is an element of the density matrix and

$$
J_{i j}=\sum_{k, l} R_{k l}(i j, k l),
$$




$$
\begin{aligned}
K_{i j} & =\sum_{k, l} R_{k l}(i k, l j), \\
(p q, r s) & \equiv\left\langle\varphi_{p}(1) \varphi_{q}(1)\left|1 / r_{12}\right| \varphi_{r}(2) \varphi_{s}(2)\right\rangle, \\
h_{i j} & =\left\langle\varphi_{i}(1)|h(1)| \varphi_{j}(1)\right\rangle .
\end{aligned}
$$

Now we expand $F_{i j}$ in Taylor's series about an arbitrary value of the density, say $R^{0}$, and we obtain

$$
F_{i j}=F_{i j}^{0}+\sum_{k} \sum_{l} W_{i j k l}\left(R_{k l}-R_{k l}^{0}\right)
$$

with

$$
\left.W_{i j k l} \equiv \partial F_{i j} / \partial R_{k l}\right]_{R_{0}}=(i j, k l)-\frac{1}{2}(i k, l j) .
$$

Obviously the expansion (7) only involves first order terms because the Fock matrix element $F_{i j}$ is a linear function of the elements $R_{k l}$ through Equation (2). Equation (7) is our master equation in the following development. We shall apply the Hückel approximation for the Hamiltonian matrix elements, i.e. $F_{i j}=0$ for $i$ and $j$ nonbonded. Moreover we shall restrict ourselves to the case of homomolecules. This restriction does not modify the general results.

a) Omega-Technique: For the constant term $F_{i j}^{0}$ we set

$$
F_{i j}^{0}= \begin{cases}\alpha & i=j \\ \beta & i \text { bonded to } j \\ 0 & \text { in all other case. }\end{cases}
$$

The most successful application of the semiempirical methods is for alternant hydrocarbons. So we choose for $R^{0}$ the values corresponding at that class of molecules [11], i.e.

$$
R_{k l}^{0}= \begin{cases}q_{l}=1 & k=l, \\ p_{k l}=1 & k \text { bonded to } l \\ 0 & \text { in all other case }\end{cases}
$$

Among the integrals $(i j, k l)$ the monocentric ones $(i i, i i)$ are those which have the highest values. If we retain only such kind of integrals, then

$$
W_{i j k l}= \begin{cases}\frac{1}{2}(i i, i i) & i=j=k=l, \\ 0 & i \neq j .\end{cases}
$$

By replacing the electron repulsion integral $(i i, i i)$ by $-2 \omega \beta$, where $\omega$ is the omega parameter, we obtain

$$
\begin{aligned}
& F_{i i}=\alpha+\omega \beta\left(1-q_{i}\right), \\
& F_{i j}=\beta ; \quad i \neq j
\end{aligned}
$$

which are the well-known expressions for the Hamiltonian matrix elements in the omegatechnique.

b) Double Omega-Technique: When we retain the integrals $(i i, j j)$ as well as $(i i, i i)$ we have

$$
W_{i j k l}=\left\{\begin{aligned}
\frac{1}{2}(i i, i i) & i=j=k=l, \\
(i i, j j) & i=j ; k=l ; i \neq k, \\
-(i i, j j) & i=k ; l=j ; i \neq j .
\end{aligned}\right.
$$

If the two-centre two electron repulsion integral $(i i, j j)$ is taken to be equal to $2 \beta \omega^{\prime}$, with $\omega^{\prime}$ a second omega parameter, we get

$$
\begin{aligned}
& F_{i i}=\alpha+\omega \beta\left(1-q_{i}\right)-\sum_{\substack{j \text { bonded } \\
\text { to } i}} 2 \omega^{\prime} \beta\left(1-q_{j}\right), \\
& F_{i j}=\beta+\omega^{\prime} \beta\left(1-p_{i j}\right) ; \quad i \neq j
\end{aligned}
$$

which are the formulas given by Mathur et al. [5] for the Hamiltonian matrix elements in their double omega-technique.

c) Generalized Omega-Technique: By a straightforward extension of the above treatment we can retain the hybrid integrals $(i j, k l),(i i, k l)$, $(i k, l i)$, etc. Under this assumption the following generalized omega- technique formulas are obtained

$$
\begin{gathered}
F_{i i}=\alpha+\omega \beta\left(1-q_{i}\right)-\sum_{\substack{j \text { bonded } \\
\text { to } i}} 2 \omega^{\prime} \beta\left(1-q_{j}\right) \\
+\sum_{\substack{r \text { bonded } \\
\text { to } i}} \omega^{\prime \prime} \beta\left(1-p_{i r}\right), \\
F_{i j}=\beta+\omega^{\prime} \beta\left(1-p_{i j}\right)+\sum_{\substack{r \text { bonded } \\
\text { to } i, j}} \omega^{\prime \prime \prime} \beta\left(2-p_{i r}-p_{j r}\right) \\
+\omega^{\prime \prime \prime \prime} \beta\left(2-q_{i}-q_{j}\right),
\end{gathered}
$$

where the omega parameters $\omega^{\prime \prime}, \omega^{\prime \prime \prime}$, and $\omega^{\prime \prime \prime \prime}$ are related to the hybrid integrals in a similar fashion as in the previous cases.

\section{Numerical Results and Conclusions}

To allow a direct comparison, the same molecules as those chosen by Mathur et al. [5] have been calculated with Equations (15). The values of the parameters have not been optimized, so we use for $\omega$ and $\omega^{\prime}$ the same values as those proposed by Mathur et al. $\left(\omega=1.4\right.$ and $\left.\omega^{\prime}=-0.6\right)$ and we set arbitrarily $\omega^{\prime \prime}=\omega^{\prime \prime \prime}=\omega^{\prime \prime \prime \prime}=0.1$. In Tables $1-3$ the theoretical results are presented as well as those calculated by other workers and the experi- 
Table 1. Calculated and observed bond lengths.

\begin{tabular}{|c|c|c|c|c|c|c|}
\hline \multirow{2}{*}{$\begin{array}{l}\text { Bond } \\
r-s\end{array}$} & \multirow{2}{*}{$\begin{array}{l}\text { Bond } \\
\text { order } p_{\mathrm{rs}}\end{array}$} & \multicolumn{5}{|c|}{ Bond length $(\AA) l_{\mathrm{rs}}$} \\
\hline & & $\mathrm{PPP}^{\mathrm{a}}$ & $\mathrm{SPO}^{\mathrm{a}}$ & $\mathrm{TPOT}^{\mathrm{b}}$ & $\begin{array}{l}\text { Experimental } \\
\text { values }\end{array}$ & $\begin{array}{l}\text { Present } \\
\text { calculations }\end{array}$ \\
\hline \multicolumn{7}{|c|}{ Benzene } \\
\hline $1-2$ & 0.667 & 1.393 & 1.393 & 1.399 & $1.397^{\mathrm{d}}$ & 1.400 \\
\hline \multicolumn{7}{|c|}{ Butadiene } \\
\hline $1-2$ & 0.967 & 1.350 & 1.345 & 1.345 & $1.344^{\mathrm{e}}$ & 1.350 \\
\hline $2-3$ & 0.256 & 1.456 & 1.464 & 1.470 & $1.464^{\mathrm{e}}$ & 1.468 \\
\hline \multicolumn{7}{|c|}{ Trimethylene } \\
\hline $1-2$ & 0.911 & 1.349 & 1.346 & 1.351 & $1.343^{f}$ & 1.360 \\
\hline $2-3$ & 0.287 & 1.463 & 1.469 & 1.473 & $1.453^{\mathrm{f}}$ & 1.463 \\
\hline \multicolumn{7}{|c|}{ Naphthalene ${ }^{g}$} \\
\hline $1-2$ & 0.806 & 1.373 & 1.396 & 1.378 & 1.363 & 1.377 \\
\hline $1-9$ & 0.484 & 1.421 & 1.426 & 1.428 & 1.421 & 1.430 \\
\hline $2-3$ & 0.497 & 1.416 & 1.422 & 1.424 & 1.415 & 1.428 \\
\hline $9-10$ & 0.634 & 1.403 & 1.396 & 1.413 & 1.418 & 1.405 \\
\hline \multicolumn{7}{|c|}{ Anthracene } \\
\hline $1-2$ & 0.856 & 1.369 & 1.362 & 1.370 & 1.366 & 1.369 \\
\hline $2-3$ & 0.416 & 1.422 & 1.432 & 1.435 & 1.419 & 1.441 \\
\hline $1-13$ & 0.409 & 1.428 & 1.437 & 1.439 & 1.434 & 1.443 \\
\hline $13-14$ & 0.556 & 1.412 & 1.409 & 1.426 & 1.428 & 1.418 \\
\hline \multicolumn{7}{|c|}{ Tetracene } \\
\hline $1-2$ & 0.877 & 1.364 & 1.358 & 1.367 & 1.385 & 1.365 \\
\hline $2-3$ & 0.377 & 1.429 & 1.440 & 1.440 & 1.479 & 1.448 \\
\hline $1-13$ & 0.374 & 1.436 & 1.445 & 1.444 & 1.431 & 1.448 \\
\hline $13-14$ & 0.493 & 1.421 & 1.422 & 1.434 & 1.439 & 1.438 \\
\hline $12-13$ & 0.709 & 1.390 & 1.385 & 1.395 & 1.398 & 1.393 \\
\hline $12-15$ & 0.540 & 1.412 & 1.415 & 1.418 & 1.409 & 1.421 \\
\hline $15-16$ & 0.538 & 1.419 & 1.413 & 1.431 & 1.478 & 1.421 \\
\hline
\end{tabular}

a M. J. S. Dewar and C. J. Gleicher, J. Amer. Chem. Soc. 87, 685, 692 (1965). b Ref. [5], Table II.

c $l_{\mathrm{rs}}(\AA)=1.51017-0.16469 p_{\mathrm{rs}}$. $\quad$ d B. P. Stoicheff, Can. J. Phys. 32, 339 (1954).

e Ar. H. Cole, G. M. Mohay, and G. A. Osborne, Spectrochim. Acta 23 A, 909 (1967).

f E. A. Dorko, J. L. Henscher, and S. H. Bauer, Tetrahedron 24, 2425 (1968).

g Experimental values for naphthalene, anthracene and tetracene were taken from Ref. ${ }^{a}$.

Table 2. Ionization potential for polycenes $\left(m_{j}=\mathrm{HOMO}\right.$ energy in units of $\beta$ ).

\begin{tabular}{lllll}
\hline Molecule & CNDO/S 2 ${ }^{\mathrm{a}}$ TPOT $^{\mathrm{b}}$ & $\begin{array}{l}\text { Experi- } \\
\text { mental } \\
\text { values }^{\mathrm{c}}\end{array}$ & $\begin{array}{l}\text { Present } \\
\text { calcula- } \\
\text { tion }\end{array}$ \\
\hline Benzene & $\mathbf{9 . 2 4}$ & 9.23 & 9.24 & 9.16 \\
Naphthalene & 8.06 & 8.15 & 8.15 & 8.22 \\
Anthracene & 7.40 & 7.44 & 7.40 & 7.50 \\
Tetracene & 7.00 & 6.98 & 7.01 & 6.97 \\
Pentacene & 6.74 & 6.65 & 6.64 & 6.58 \\
\hline
\end{tabular}

a See comments below Table III in [5]. - b Reference [5].

c N. O. Lipari and C. B. Duke, J. Chem. Phys. 63, 1768 (1975).

d I.P. $(\mathrm{eV})=4.315+5.1955 m_{j}$.
Table 3. Position of $\mathrm{Vl} \leftarrow \mathrm{N}$ electronic transition for polyacenes $(\Delta m=$ HOMO-LEMO energy in units of $\beta$ ).

\begin{tabular}{|c|c|c|c|c|}
\hline \multirow{2}{*}{$\begin{array}{l}\text { Molecule } \\
\text { Benzene }\end{array}$} & \multicolumn{2}{|c|}{$\mathrm{CNDO} / \mathrm{S} 2^{\mathrm{a}} \mathrm{TPOT}^{\mathrm{b}}$} & \multirow{2}{*}{$\begin{array}{l}\begin{array}{l}\text { Experi- } \\
\text { mental } \\
\text { values c }\end{array} \\
48400\end{array}$} & \multirow{2}{*}{$\begin{array}{l}\text { Present } \\
\text { calcula- } \\
\text { tiond }\end{array}$} \\
\hline & 48593 & 48166 & & \\
\hline Naphthalene & 34445 & 35097 & 34700 & 36025 \\
\hline Anthracene & 26235 & 26535 & 26400 & 27353 \\
\hline Tetracene & 21119 & 20903 & 21200 & 21075 \\
\hline Pentacene & 17708 & 17448 & 17400 & 16410 \\
\hline
\end{tabular}

a Table IV in [5]. - b Table IV in [5].

c S. C. Mathur and D. C. Singh, Ind. J. Pure Appl. Phys. $8,788(1970)$

d $E_{V l \leftarrow N}\left(\mathrm{~cm}^{-1}\right)=-5851.4+\Delta m$ 3C629.0. 
mental ones. The numerical values show that the Generalized Omega-Technique predicts bond lengths, ionization potentials and positions of $\mathrm{Vl} \leftarrow \mathrm{N}$ electronic transitions of poliacenes in very good agreement with the experiment.

The derivation presented for the different omegalike methods is general in the sense that the Hamiltonian matrix elements are deduced from the master equation (14), which is completely valid in the context of the Hartree-Fock formalism. Thus the omega and double omega techniques emerge in a natural way insofar as we retain a certain class of two-centre two electron repulsion integrals and neglect others. From an obvious extension, a generalized omega-technique is derived, where both diagonal and non-diagonal Hamiltonian matrix elements depend on atomic charges and bond orders. There exists a certain arbitrary character for

[1] R. Pariser and R. G. Parr, J. Chem. Phys. 21, 466, 767 (1953).

[2] J. A. Pople, Trans. Faraday Soc. 49, 1375 (1953).

[3] G. W. Wheland and D. E. Mann, J. Chem. Phys. 17, 264 (1949).

[4] E. A. Castro and F. M. Fernández Rev. Roum Chim. (in press)

[5] S. C. Mathur, D. C. Singh, B. Kumar, and S. Mitra, Int. J. Quantum Chem. 11, 759 (1977).

[6] S. C. Sharma, A. K. Srivastava, and B. Krishna, J. C. S. Faraday II 70, 168, 172 (1974).

[7] E. A. Dorko, H. P. Nielson, and W. C. Bahr, Theor. Chim. Acta 14, 357 (1969). the choice of the matrix $F^{0}$. The formula for the matrix elements $F_{i j}^{0}$ is different in each method. So, for the omega-technique

$$
\begin{aligned}
& F_{i i}^{0}=h_{i i}+\frac{1}{2}(i i, i i), \\
& F_{i j}^{0}=h_{i j},
\end{aligned}
$$

whereas for the double omega-technique

$$
\begin{aligned}
& F_{i i}^{0}=h_{i i}+\frac{1}{2}(i i, i i)+\sum_{\substack{j \text { bonded } \\
\text { to } i}}(i i, j j) . \\
& F_{i j}^{0}=h_{i j}-\frac{1}{2}(i i, j j) ; \quad i \neq j .
\end{aligned}
$$

Comparing $(16)-(17)$ with $(18)-(19)$ we see that the matrix $F^{0}$ is not univocally defined. But this is not important in this kind of methods, because $F_{i i}^{0}$ is chosen as the zero for the energy scale, and $F_{i j}^{0}$ is taken as the unit of energy.

[8] L. K. Huy and W. Forst, Theor. Chim. Acta 16, 393 (1970).

[9] W. Grundler, Monatsh. Chem. 101, 1362 (1970).

[10] M. Hida, Memoirs of Faculty of Technology, Tokyo Metropolitan University, Tokyo 24, 2209 (1974).

[11] A. Streitwieser, Jr., Molecular Orbital Theory for Organic Chemists, John Wiley, New York 1961.

[12] R. L. Flurry, Jr., Molecular Orbital Theories of Bonding in Organic Molecules, Marcel Dekker, New York 1968.

[13] E. A. Castro and F. M. Fernández Bol. Soc. Quimica (Peru) (in press).

[14] S. P. Gupta and B. Krishna, J. Phys. B 5, 1101 (1972).

[15] K. F. Freed, Mod. Theor. Chem. 7, 201 (1977). 